\title{
LA NEGOCIACIÓN EN TIEMPOS DEL COVID-19
}

\author{
FRANK GARCÍA ASCENCIOS* \\ Universidad de Lima, Lima, Perú \\ frankgarciaascencios@gmail.com
}

Recibido: 28/11/2020 Aprobado: 5/12/2020

doi: https://doi.org/10.26439/iusetpraxis2021.n052.4991

\begin{abstract}
RESUMEN. Sobre la base de los conceptos de negociar y regatear, el artículo discute la afirmación de que "diariamente negociamos". En tal escenario, se pretende mostrar el traslado de una negociación por posiciones a una por intereses, en la línea del Harvard Negotiation Project, a fin de distinguir en el proceso de negociación entre las personas y el problema. De esta manera, se pueden plantear opciones de beneficio mutuo, centrándose en criterios objetivos para ganar en resultados y empatía. El actual contexto del COVID-19, y el entorno de tecnología que urge y supedita, muestra con mayor claridad la necesidad de contar con nuevas habilidades digitales para abordar una negociación. De igual modo, en la conciliación extrajudicial, el conciliador tiene como reto adecuarse al uso de la plataforma virtual y propender al desarrollo de estas habilidades digitales, dentro de un contexto sancionador por parte del Ministerio de Justicia y Derechos Humanos.
\end{abstract}

PALABRAS CLAVE: conciliación extrajudicial / COVID-19 / empatía / Ministerio de Justicia y Derechos Humanos / MARCS / negociación

\footnotetext{
* Abogado y magíster en Derecho Empresarial por la Universidad de Lima (Perú). Máster en Derecho (LLM) por la Universidad de Hawái en Mānoa (Estados Unidos). Máster en Seguros y Gerencia de Riesgos por la Universidad Pontificia de Salamanca (España). Profesor en la Universidad de Lima.
} 


\section{NEGOTIATIONS IN TIMES OF COVID-19}

ABSTRACT. Based on the concepts of "negotiate" and "haggle", this article discusses the claim that "we daily negotiate". In such a scenario, the author intends to show the transfer from a position-based to an interest-based negotiation, according to the Harvard Negotiation Project, in order to distinguish the persons from the problem in the negotiation process. Thus, this may lead to raise mutual benefit options by focusing on objective criteria to achieve better results and empathy. The current COVID-19 context, as well as the urgent and contingent technological environment, clearly shows the need for new digital skills to tackle a negotiation. Similarly, in cases of extrajudicial conciliation, the conciliator faces the challenge of adapting to virtual platforms and promoting digital skills within sanction regimes administered by the Ministry of Justice and Human Rights.

KEYWORDS: extrajudicial conciliation / COVID-19 / empathy / Ministry of Justice and Human Rights / alternative dispute resolution / negotiation 


\section{INTRODUCCIÓN}

Los tiempos no son los mismos. Todo ha cambiado. Esta época de pandemia ha generado que cada uno se adecúe a la "nueva normalidad" en la forma de vivir en sociedad y también de ofrecer servicios legales.

El hecho de adaptarse a esta realidad no significa que se brinde algo nuevo del todo: no es que un profesor de historia tenga que aprender otra vez historia universal, no es que un arquitecto tenga que aprender a diseñar. Un abogado tampoco tendrá que volver a aprender cómo interpretar las leyes, se encuentren estas en un papel o en un archivo digital. Es lejano a la realidad sostener que debe aprenderse nuevamente lo ya conocido; lo que hay que hacer es cambiar y adecuarse al presente.

La negociación es el arte de poder conversar y arribar a acuerdos sensatos, eficientes y que no deterioren la relación'. Al ser la negociación una materia donde el diálogo es el principal instrumento, no hay duda de que la virtualidad influirá con fuerza. Tal escenario no será un problema para las negociaciones internacionales o las nacionales que involucran agentes que ya venían procediendo en esta era digital, pero sí tendrá un efecto en las negociaciones del día a día entre agentes cotidianos, los cuales buscan negociar sobre los contratos civiles, temas familiares y laborales, entre otros.

El presente artículo se enfoca en estos casos cotidianos de negociación de menor suma económica, pero trascendentales para lograr un acceso a una justicia alternativa en las grandes mayorías. En estos casos, la asistencia presencial ha sido considerada como mandatoria, debido a que se busca arribar a un acuerdo. Las sesiones virtuales son una característica ajena a la costumbre doméstica.

Se inicia abordando la negociación, sus principales características y el impacto de la pandemia del COVID-19 en este mecanismo alternativo de solución de conflictos. Asimismo, se aprovecha la oportunidad para reflexionar sobre el papel que ha tenido la conciliación extrajudicial, como negociación asistida, en estos tiempos de pandemia, y su camino a la virtualización forzada.

\section{NEGOCIACIÓN}

\section{Definición}

Negociación es una palabra que se emplea continuamente. Incluso se dice que uno negocia a diario, cuando se adquiere algún bien o al recibir un servicio. Nada más

1 Fisher, Ury y Patton (1992) afirman que "cualquier método de negociación debe juzgarse conforme a tres criterios: debe conducir a un acuerdo sensato, si el acuerdo es posible; debe ser eficiente; $y$ debe mejorar, o por lo menos no deteriorar, la relación entre las partes" (p. 7). 
inexacto ${ }^{2}$. Si se negociara cada día, todos serían negociadores expertos, con habilidades blandas y no se tendría el alto índice de judicialización que existe en el país.

No debe confundirse el hecho de negociar con el de regatear. El regateo solo se enfoca en el precio, exclusivamente en un aspecto monetario; no existe un diálogo donde se busque encontrar los intereses de la otra parte y, paradójicamente, ni el que regatea conoce lo que realmente necesita. El diccionario de la Real Academia Española (2020) señala que regatear es "debatir el precio de algo puesto en venta". En cambio, el término negociar puede definirse como "tratar asuntos públicos o privados procurando su mejor logro". A partir de ello, se observa que ser buenos regateadores no significa ser buenos negociadores.

La negociación es el arte de dialogar y escuchar verdaderamente a la otra persona. Es entender que el objeto no es alcanzar un acuerdo en el que uno sea el beneficiado. El acuerdo es importante, pero de nada sirve dicho pacto si es incumplido y, posteriormente, genera que se recurra a la vía judicial para resolver el conflicto surgido.

Entonces, la negociación debe concluir con un acuerdo satisfactorio para ambas partes, lo cual significa que exista un criterio de equidad o justicia. Un criterio justo no será incumplido y garantiza el éxito de la negociación. En tal sentido, Shell (2005) señala que "la negociación avanza mediante una forma de comunicación prudente y cooperativa. Y la negociación sigue, por lo general, un camino reconocible que consta de cuatro pasos: preparación, intercambio de información, la negociación propiamente dicha y el compromiso" (p. 5).

La negociación es comunicación mutua, la cual debe ser prudente, ya que ambas personas son seres emocionales y un término no adecuado en el diálogo puede llevar a la ruptura. Asimismo, la colaboración es necesaria, pues el aprovechamiento unilateral es iluso. Ambas partes serán las beneficiadas, de eso no existe la menor duda.

La negociación es sinónimo de esfuerzo tanto en la etapa previa como durante el mismo momento de la negociación. Necesita de preparación, de saber escuchar, de empatía, de ser imaginativo para proponer opciones de solución, de ser razonables y objetivos.

2 Hernández (2011) cuestiona la presunción de que se negocia todos los días, pues afirma que "[...] Negociamos todos los días, con nuestra pareja, clientes, proveedores... Así comienzan muchos libros sobre negociación. ¿Está seguro de que negociamos todos los días? Si todos lo hiciéramos, acudiríamos a negociar con menos ansiedad y, sin embargo, lo hacemos con desazón e incertidumbre. ¿Cómo es posible?" (p. 7). 


\section{De la negociación por posiciones a la negociación por intereses (Harvard)}

De manera clásica, la negociación ha sido concebida como un trato por posiciones. Posteriormente, se ha pasado a una negociación por intereses o colaborativa. Este cambio no es menor, debido a que ahora la definición misma de negociación está vinculada con la satisfacción de intereses.

La negociación por posiciones centra el diálogo en aspectos superficiales. No existe un conocimiento de por qué se quiere algo. El trabajador busca un aumento de su remuneración, no hay mayor análisis de por qué quiere el incremento salarial. La exesposa pide un incremento de pensión de alimentos, pero se observa que no se produce un incremento de gastos del menor. El inquilino moroso dice que no pagará las rentas impagas; el propietario busca el pago inmediato de rentas y desalojarlo del inmueble.

La negociación basada en posiciones no lleva a ningún análisis o razonamiento de por qué se tiene determinada posición. No se analiza que los hijos del trabajador accederán a un instituto o universidad el próximo año. Ese es el motivo por el que busca un incremento de remuneración. La exesposa pide un incremento de pensión de alimentos, a pesar de no existir más gastos por parte del menor. Se conversa con la señora y, finalmente, expresa que su exesposo no visita al menor. Ese el motivo por el que pide más pensión, por la amargura contra su expareja. El inquilino moroso tiene tres hijos y su esposa ha perdido el trabajo, no tiene liquidez, no paga las rentas, debido a que, de hacerlo, no podrá pagar la pensión escolar de sus hijos.

La negociación por posiciones genera acuerdos insensatos, ineficientes y deteriora la relación. No es adecuado arribar a acuerdos sin comprender la conducta de las personas frente al conflicto, pues, en tal caso, se trata de acuerdos ciegos y se mira por encima el proceder de la contraparte, sin entender su conducta. El no conocer las razones y motivaciones del interlocutor genera que estos acuerdos no perduren en el tiempo, generalmente, son inaplicados y, lo peor, perjudica las relaciones entre ambos sujetos.

Entonces, la negociación según principios o colaborativa surge como una respuesta natural a cómo se debe negociar. El diálogo no debe ser débil o fuerte, sino que las palabras deben orientarse a descubrir las motivaciones de por qué se pide algo, es decir, a buscar los intereses de las personas. En otras palabras, para saber lo que realmente se quiere, hay que conocer sus pensamientos, temores y necesidades. El escuchar es una pieza fundamental para lograr este objetivo.

Si se procediera según principios o colaboración, entonces, la empresa empleadora del trabajador que pide aumento de sueldo podría ofrecer descuentos o becas para estudiar en la universidad de propiedad de uno de sus socios. De igual modo, el exesposo pensaría en el daño que puede estar causando al menor al no visitarlo como padre, 
podría cambiar su conducta y su exesposa estaría tranquila, sin solicitar incremento de pensión. Finalmente, si el propietario conociera de los problemas que afronta su inquilino, tendría una visión amplia del conflicto, e incluso podría llegar a un primer acuerdo para recuperar la posesión del inmueble, a fin de arrendarlo a un nuevo inquilino y seguir percibiendo ingresos.

\section{Cuatro puntos definen la negociación por intereses (Harvard)}

El año 1979, se creó el Harvard Negotiation Project. Posteriormente, los profesores Roger Fisher y William Ury publican Getting to yes. Negotiating an agreement without giving in, un best seller que propone la negociación por intereses o según principios o colaborativa o de méritos. Este libro marca un antes y un después en el estudio y desarrollo práctico de la negociación, convirtiéndose en el manual básico de quien desea negociar. En el pensamiento americano, la negociación se concibe como instrumento necesario para resolver conflictos contractuales o mercantiles ${ }^{3}$, áreas donde se ha aplicado estos puntos cardinales de la negociación por intereses.

La práctica nacional adopta estos elementos para abordar toda negociación. De igual modo, lo hace en la mediación o conciliación, donde el conciliador domina estos elementos. No obstante, también es cierto que se está incrementando el uso de mediación transformativa ${ }^{4}$ en conflictos familiares, escolares u otros vinculados.

Es forzoso precisar que la negociación colaborativa también busca que las relaciones perduren, de ninguna manera pierde ese horizonte, porque comprende que los buenos acuerdos deben mantener necesariamente la relación entre las personas. La negociación colaborativa no solo se enfoca en satisfacer intereses, sino también en las emociones de las personas, en la consideración de que se genere una solución que ambas partes estimen justa o adecuada y que dicha relación o vínculo deba continuar en el tiempo.

El libro Getting to yes. Negotiating an agreement without giving in (Fisher, Ury y Patton, 1992) desarrolla cuatro puntos centrales para construir la denominada negociación al estilo Harvard.

3 Otra área donde se aplica la negociación es la de los secuestros, en la cual se enfoca en lo emocional sobre lo racional. Esta es una forma distinta de abordar la negociación, ya que se logra una empatía táctica con el secuestrador a fin de lograr la liberación de rehenes. Un libro recomendable sobre esta temática es el de Voss (2016).

4 La negociación transformativa tiene por objeto transformar a las personas, no el arribar a un acuerdo. Este tipo de negociación se enfoca en convertir a las personas en generosas, a fin de que puedan reflexionar sobre su conducta y los efectos generados en la contraparte. Se busca cambiar la vida de las personas. El texto que desarrolla esta teoría es el de Bush y Folger (1996). 


\section{Separar a las personas del problema}

El ser humano es un ser emotivo, pues con frecuencia guía su conducta con base en emociones. Se afirma que todos cuentan en su ser con un hombre de Cromañón ${ }^{5}$; es decir, en una negociación acuden dos seres racionales, pero, a la vez, cada uno es acompañado por su ser irracional.

La irracionalidad lleva a pensar que el conflicto se produce por la otra persona y, por tanto, se ataca a la contraparte. Sin embargo, aquello limita la visión del problema, pues no se centra en este y, entonces, se pierde energías en agredir a la otra persona, lo cual deteriora la relación y genera un conflicto que no puede ser solucionado mediante un acuerdo.

El individuo es un ser emocional. Es complicado dejar de lado lo emocional, pero hay que hacerlo, con el objeto de concentrarse en comprender y analizar cada problema en particular, sin que las emociones se conviertan en un limitante para ello.

\section{Concentrarse en intereses, no en posiciones}

Para solucionar el problema, uno no puede quedarse en las palabras exteriorizadas por la otra persona. Se expresa lo que se quiere, pero no el porqué de sus motivaciones, razones y necesidades. En otras palabras, solo escuchamos las posiciones y no los intereses, lo cual es un grave error.

No puede arribarse a una solución eficiente si es que no hay un enfoque en los intereses, puesto que la opción de solución más razonable debe satisfacer las necesidades de ambas partes. Por ejemplo, Juan está interesado en comprar un vehículo usado, encuentra uno, pero no puede pagar el precio. El vendedor le pregunta qué desea del vehículo; Juan le dice que todos los días maneja desde La Molina hasta el Callao, que existe una enorme distancia y que no desea tomar transporte público por la inseguridad ciudadana. El vendedor le ofrece otro vehículo a Juan, con un motor ahorrador de gasolina, que posibilitará gastar menos en combustible y a un menor precio. Ambas partes arriban a un acuerdo en el precio del nuevo vehículo.

El descubrir los intereses no es sencillo. Hay que escuchar y preguntar. Debe formularse las siguientes preguntas: ¿por qué?, ¿para qué?, ¿es seguro?, a fin de descubrir las necesidades de la otra persona. Es elemental tener presente que, mientras se logre satisfacer las necesidades de la contraparte, será factible arribar a un acuerdo. Asimismo, uno debe respetar las motivaciones, razones o necesidades de la otra parte.

5 "Cuando negociamos actuamos como si alguien nos obligara a obrar de una manera emotiva e instintiva. Ese alguien, presente en cada uno de nosotros, guarda parte de nuestros instintos y hábitos que dirigen nuestro comportamiento. Es el hombre de Cromañón que todos llevamos dentro. Es nuestro perpetuo acompañante que se niega a ser controlado, porque atiende más a los instintos y las emociones que a su razón" (Hernández, 2011, p. 19). 


\section{Inventar opciones de mutuo beneficio}

Las opciones son las posibilidades que se presentan dentro de la mesa de negociación. Estas posibilidades no tienen que ser sesgadas; es decir, no deben estar orientadas exclusivamente a favorecer a una sola persona. Si aquello es así, es más que seguro que terminará en los tribunales. Las posibilidades han de tener una visión de mutuo beneficio si es que se busca alcanzar relativamente algún éxito en la negociación.

La clave para conseguir opciones de mutuo beneficio es conocer los intereses del otro. Para lograr ello, hay que descubrir los intereses, tanto los propios como los del sujeto que se tiene al frente. Es difícil inventar opciones de mutuo beneficio si es que no se ha escuchado a la otra persona.

La lluvia de ideas es una herramienta que ayuda a generar diversos criterios que coadyuven a solucionar el problema. El plantear ideas u opciones resulta conveniente para contar con varias posibilidades. Después, se analiza cada una de las alternativas para considerar su viabilidad. Al final, se elige la mejor opción para ambas partes.

Debe tenerse presente que fuera de la mesa de negociación se encuentran alternativas, posibilidades que surgen más allá de la negociación, pero que sirven como guía para determinar si se arriba a un acuerdo. Uno no puede actuar de forma ineficiente; debe mirar más allá de la mesa de negociación a fin de determinar si puede arribar a un acuerdo más eficiente afuera.

En ese contexto, Harvard desarrolla la noción de BATNA (best alternative to a negotiated agreement) o MAAN (mejor alternativa al acuerdo negociado). La MAAN permite que no se dependa exclusivamente del acuerdo al que se puede arribar en la negociación, sino mirar afuera de la conversación, apreciar si puede alcanzarse un acuerdo que satisfaga los intereses.

La MAAN es lo mínimo que se está dispuesto a recibir en la negociación, debido a que afuera se puede recibir ese mínimo. Es importante tener en consideración que "si ambas partes tienen una MAAN atractiva, es posible que el mejor resultado de la negociación, para ambas partes, sea no llegar a un acuerdo" (Fisher, Ury y Patton, 1992, p. 54).

\section{Centrarse en criterios objetivos}

El acuerdo al que arriben las partes necesariamente debe seguir criterios de naturaleza objetiva. Dicho de otro modo, los números, estadísticas, encuestas, normas y precedentes deben guiar el acuerdo. Nadie desconoce un acuerdo sustentado en criterios objetivos.

Por ejemplo, María solicita una indemnización producto de una mala operación en una clínica. Ella no puede caminar con normalidad, ha sido afectada en la cadera. Ella 
no tiene trabajo estable, no tiene ingresos durante los últimos años. Pide un millón de soles de indemnización. La clínica consulta a sus abogados sobre cuál es el monto indemnizatorio que se otorga en la vía judicial en casos similares; ellos le dicen que no es ni la cuarta parte de lo que se pretende. María también entiende aquello. Frente a este escenario, está dispuesta a llegar a un acuerdo con la clínica, pero por un monto considerablemente menor al millón de soles. En este supuesto, el acuerdo ha sido guiado por criterios objetivos: la casuística de montos indemnizatorios en el Poder Judicial.

Los buenos acuerdos siguen criterios objetivos. Por el contrario, cuando uno se aparta de la objetividad, se tienen acuerdos que posteriormente son incumplidos con el argumento de resultar abusivos e injustos. En este escenario, si hay incumplimiento, pierden todos.

Estos cuatro puntos esenciales fueron desarrollados por la negociación colaborativa o Harvard. De estos puntos cardinales se desprende lo que se conoce como los siete elementos de la negociación Harvard: relación, comunicación, intereses, opciones, legitimidad (criterios), alternativas y compromisos.

Relación, porque es importante que el acuerdo permita mantener la relación comercial. Comunicación, pues el negociador debe escuchar y ser empático. Intereses, ya que deben descubrirse las necesidades de las personas. Opciones, es decir, las posibilidades dentro de la negociación. Legitimidad, que es otorgada al acuerdo por los criterios objetivos. Alternativas, que son las posibilidades afuera de la mesa de negociación. Compromiso, que son los acuerdos a los que se ha arribado. Este no es un texto para desarrollar los elementos; se prefiere que el lector acuda a la fuente, el libro Getting to yes. Sin embargo, no queda la menor duda de que la comprensión de estos conceptos no se da en una simple lectura del texto; por ello, es importante haber brindado algunas reflexiones e ideas centradas en un mayor entendimiento de la negociación.

\section{Empatía}

La empatía es el arte de ponerse en el lugar del otro. No es sencillo hacerlo. Por naturaleza, al ser humano primero le importa lo que pase con él y luego los demás. Para decirlo en palabras sencillas, para usted primero será usted, luego el otro. Es la naturaleza humana.

Goleman (2019, p. 4) clasifica tres tipos de empatía: cognitiva, emocional y el interés empático. La primera se refiere a la capacidad para entender la perspectiva del otro. La segunda manifiesta la capacidad para sentir lo que siente el otro. El tercero es la capacidad para comprender lo que la otra persona necesita de uno. 
Si no tiene empatía, difícilmente podrá arribar a un acuerdo ${ }^{6}$; no habrá un diálogo verdadero. Las emociones rodean cada conflicto $y$, si uno ni siquiera puede ponerse en lugar de otro, la conversación será un fracaso. Las acciones de las personas están motivadas por razones poderosamente importantes para cada uno; si el otro no entiende esa razón, no habrá acuerdo. Ponerse en lugar del otro no significa estar de acuerdo con lo que piense.

Es difícil que las personas puedan tener estas habilidades. Si uno está en el conflicto, es complicado que busque comprender las acciones del otro, o que busque una solución beneficiosa para ambos, o que escuche y trate de encontrar los intereses de la otra parte. En ese contexto, se han propuesto otros mecanismos alternativos de solución de conflictos, tales como la mediación y la conciliación, donde un tercero ajeno al conflicto es quien goza de estas habilidades para acercar a las partes y lograr que arriben a un acuerdo que posibilite soluciones.

Estas habilidades son aplicadas por terceros, dígase conciliadores, quienes guiarán el diálogo entre las partes. Su propia naturaleza, al no ser parte del conflicto, les confiere mayor facilidad para poder llevar adelante estas habilidades. La labor del conciliador es fundamental dentro del ambiente de cultura de paz.

\section{LA NEGOCIACIÓN DIGITAL}

Además de todas las habilidades que se debe tener como buenos negociadores, se ha sumado ahora un nuevo elemento, lo virtual. El uso de la tecnología para llevar adelante las negociaciones es reciente en el país, tal vez forzado por la pandemia del COVID-19. La realidad ha privilegiado lo presencial sobre lo virtual. Si bien aquello no es un problema para las negociaciones entre representantes de empresas o agentes que ya estuvieron empleando medios digitales, también es verdad que no debe dejarse de lado los casos de menor cuantía. Este aspecto es importante para descongestionar el sistema nacional de justicia y porque debe percibirse que el Estado propicia mecanismos alternativos para que sean las mismas personas quienes pongan fin a sus conflictos.

En tal contexto, resulta un desafío la negociación digital, sobre todo porque ahora no solo hay que centrarse en las habilidades ya desarrolladas, sino también en manejar adecuadamente las plataformas virtuales. Hoy es una imperiosa necesidad mantener en condiciones óptimas los equipos de cómputo, así como prestar mayor importancia a la calidad de conectividad de internet. Antes uno se preparaba exclusivamente para el caso, ahora debe dedicar tiempo a probar con anticipación la plataforma virtual. Ningún

6 "La empatía amplía el conjunto de opciones que tenemos para resolver el conflicto. Cuanto mejor comprendamos la perspectiva de la otra parte, más probabilidades tendremos de encontrar una solución" (Malhotra, 2016, p. 207). 
error de la tecnología puede resultar una barrera para que las partes dialoguen con tranquilidad, a fin de llegar a un acuerdo.

\section{No presencia física, sino virtual}

Si bien es positivo que se puedan realizar acciones desde cualquier lugar donde uno se encuentre, gracias al soporte de la tecnología, resulta difícil pronunciarse sobre su efectividad. Sobre todo, cuando se está frente a casos familiares, laborales u otros similares, en los que el factor personal es preponderante. No por nada el carácter presencial ha sido una característica a nivel nacional. Un ejemplo es la conciliación extrajudicial, donde se ha preferido, por ley, a lo largo de los años, que la sesión de conciliación sea presencial y en un centro de conciliación físico.

El desempeño que tengan las partes en una negociación puede ser perjudicado si es que uno no tiene estas habilidades tecnológicas. Es elemental considerar que se está frente a un conflicto y, entonces, sería el colmo que el uso no adecuado de la tecnología también se convierta en una barrera para arribar a un acuerdo.

En lo que respecta a la conciliación extrajudicial, el conciliador debe gozar de habilidades tecnológicas; no hacerlo marcaría un fracaso para acercar a las partes a que puedan escucharse y descubrir sus intereses. Asimismo, ha de tener un adecuado manejo de emociones de las partes, debido a que, sumado al conflicto, ahora estas deberán contar con habilidades digitales y, si alguna de ellas no tiene paciencia, entonces, es posible que genere emociones negativas que nublen la posibilidad de continuar el diálogo. El conciliador debe guiar la sesión.

La falta de presencia física en la sesión es un reto. Frente a ello, el uso de la cámara es fundamental, pues la imagen proyecta calidad humana. Incluso las imágenes que se transmiten pueden producir percepciones positivas para que las partes sientan que este nuevo sistema permite rendir resultados positivos.

\section{Internet}

Jamás debe olvidarse el horizonte de una negociación: arribar a acuerdos sensatos, eficientes y que no deterioren la relación. Para lograr este objetivo, el tener una buena conectividad es importante. Por supuesto, internet no reemplaza las habilidades de un buen negociador, pero es un instrumento sustancial. Hoy resulta imperativo contar con un servicio de internet veloz y con equipos en condiciones óptimas. Si no se posee una conexión rápida y sin fallas, será difícil seguir adelante con la negociación, no debido a debilidades de las partes o ausencia de habilidades dentro de la negociación, sino por las falencias de la tecnología.

Si bien cada vez hay mayor conectividad, también es cierto que el Perú tiene varios problemas en cuanto a la velocidad de internet. Según el Organismo Peruano 
de Consumidores y Usuarios (Opecu), en agosto del 2020 se ha tenido velocidades de internet fijo de 35,3 megabits y móvil de 21,8 megabits, en comparación con la velocidad fija de 84,3 megabits y móvil de 34,8 megabits a nivel global. Estas cifras indican que el internet del país fijo y móvil está 58 \% y 37 \%, respectivamente, por debajo de la media mundial. Estas son cifras anuales en promedio.

Frente a esta problemática del internet en el país, se deben tomar precauciones antes del inicio de la sesión de negociación. Así, por ejemplo, es preferible conectarse mediante cable de red, no por wifi. De igual modo, antes de iniciar la sesión debe evaluarse la velocidad de la red. En la conciliación extrajudicial, el centro de conciliación es responsable de contar con una red y equipos de cómputo en óptimas condiciones, a fin de que el conciliador realice sus funciones.

\section{Redacción de acuerdos}

El acuerdo es el resultado al que se llega en una negociación, luego del diálogo fructífero de las partes. Es común que una de las partes involucradas, dentro de su proactividad, remita un borrador de acuerdo a la otra, a fin de disminuir cualquier riesgo de no llegar a concretar el pacto. Aquello es normal y es recomendable colaborar para que el procedimiento sea más sencillo.

Sobre la oportunidad para llegar a un acuerdo, es recomendable que este pueda redactarse en la misma sesión; sin embargo, también es una opción que una de las partes sea la que proyecte el acuerdo, de manera que en los próximos días se formalice. Asimismo, el uso de la tecnología es importante en este punto: no solo se trata de estar frente a una cámara dialogando con otra persona, sino que hay que redactar un acuerdo, para lo cual debe existir un dominio del correo electrónico.

En la conciliación extrajudicial, la presencia del conciliador coadyuva a solucionar estas contingencias, debido a que, por lo general, el conciliador remitirá estos acuerdos por escrito a las partes, quienes, previa lectura, brindarán su conformidad. Esta sesión guarda ciertas formalidades: la audiencia concluye en un acta de conciliación, que tiene mérito ejecutivo, conforme a la normativa vigente.

\section{Confidencialidad}

Los medios alternativos de resolución de conflictos (MARCS) son medios confidenciales por excelencia; sin embargo, el solo hecho de que las sesiones de negociación se realicen por medios tecnológicos provoca incertidumbre respecto de la grabación de la conversación por la otra parte. Aquello puede ser un problema, debido a que el diálogo y el no verse vinculado por la propuesta genera que se tenga una apertura en la negociación. No es igual negociar sabiendo que la sesión está siendo grabada a dialogar libremente. 
En adición a lo anterior, la conciliación extrajudicial sigue la esencia de la confidencialidad. Las propuestas expresadas en la conciliación no obligan a las partes. Será difícil evitar alguna grabación de contrabando de alguna de las partes, ya que no existe mayor control para evitarlo, en especial cuando en esta era de la tecnología es común grabarse, incluso sin el consentimiento de la otra persona. Por consiguiente, si bien ahora se permite que se grabe el momento de la identificación de las personas, el monólogo de apertura, la descripción de la controversia, los hechos objeto de reconvención (si hubiere) y acuerdos, o no, pero no el diálogo y las propuestas, ello será difícil de controlar o supervisar.

\section{LA CONCILIACIÓN EXTRAJUDICIAL Y EL RETO DE LA DIGITALIZACIÓN DURANTE EL COVID-19}

La conciliación extrajudicial es una institución de interés nacional regulada en el país, cuyo objetivo es promover la solución armoniosa de los conflictos. Se trata de una institución consensual y un mecanismo alternativo de solución de conflictos, donde las partes recurren a un centro de conciliación extrajudicial para encontrar una solución armoniosa.

La conciliación está regulada en la Ley de Conciliación, Ley N. 26872. La conciliación extrajudicial tiene como órgano rector al Ministerio de Justicia y Derechos Humanos (MINJUSDH), el cual mediante la Dirección de Conciliación Extrajudicial y Mecanismos Alternativos de Solución de Conflictos (DCMA) difunde la conciliación extrajudicial a nivel nacional. De igual modo, la DCMA conduce procedimientos conciliatorios, así como ejerce funciones de registro, supervisión y sanción de los operadores del sistema conciliatorio (conciliadores extrajudiciales, centros de conciliación extrajudicial, capacitadores y centros de formación y capacitación de conciliadores).

Lamentablemente, a pesar de la flexibilidad de los MARCS, la principal barrera de la digitalización de la conciliación extrajudicial ha sido la propia Ley de Conciliación, que ha obligado a acudir personalmente a un centro de conciliación para realizar las sesiones de conciliación?.

Si bien ha sido difícil la modificación de la ley ${ }^{8}$ para adecuarla a las nuevas tecnologías, lo complicado será la capacitación de los conciliadores para conocer y aplicar el nuevo sistema digital. Sobre todo, a partir de la consideración de la formalidad extrema

7 Artículo 5 del Decreto Legislativo N. ${ }^{\circ}$ 1070: “Definición. La conciliación es una institución que se constituye como un mecanismo alternativo para la solución de conflictos, por el cual las partes acuden ante un centro de conciliación extrajudicial a fin de que se les asista en la búsqueda de una solución consensual al conflicto". (Anterior a la modificación)

8 La Ley de Conciliación fue modificada por la Ley N. 31165 . Asimismo, el reglamento de la Ley de Conciliación fue modificado por el Decreto Supremo N. ${ }^{\circ}$ 008-2021-JUS. 
que se sigue en el procedimiento conciliatorio, que incluye lo relacionado con las actas de conciliación. Esta formalidad debe cumplirse a la luz de la regulación de la conciliación extrajudicial, pues, de no hacerlo, podrá caerse en vicios de invalidez del acta de conciliación. Asimismo, el conciliador y el centro de conciliación podrían ser sancionados por incumplir la Ley de Conciliación y su reglamento.

El reto de la digitalización debe llevar a reflexionar sobre dejar de lado las formalidades draconianas en la conciliación extrajudicial y repensar en colocar un mayor énfasis en la institución como tal, en lograr un diálogo con libertad de las partes, donde el conciliador no actúe con miedo a cualquier sanción administrativa por parte del MINJUSDH, sino orientado a lograr un resultado: el entendimiento de las partes del conflicto y un acuerdo que perdure a lo largo del tiempo.

En consecuencia, estoy convencido de que la conciliación virtual debe pasar más allá de la posibilidad de emplear medios tecnológicos para las sesiones conciliatorias. Se tiene que repensar en fortalecer el sistema, pero desde su misma esencia como mecanismo alternativo, no desde el ámbito sancionador, el cual no debe ser una prioridad en la conciliación extrajudicial.

El MINJUSDH ha perdido la oportunidad de repensar el sistema de conciliación extrajudicial. Ha basado la última modificación de la Ley de Conciliación en la necesidad de regular las audiencias a través de medios digitales, forzado por la pandemia del COVID-19, sin abordar la problemática del engorroso procedimiento de notificaciones a las partes, la extremada formalidad para designar un apoderado para acudir a la sesión de conciliación y la extrema sobrerregulación del procedimiento conciliatorio.

\section{CONCLUSIONES}

La negociación es un mecanismo alternativo de resolución de conflictos que permite el diálogo entre las personas, a fin de arribar a un acuerdo sensato, eficiente y que no deteriore la relación. Negociar no es lo mismo que regatear. La negociación es el arte de dialogar y escuchar a la otra persona.

No es cierto afirmar que siempre negociamos. La negociación permite tener una visión amplia del conflicto, se busca las reales necesidades de la contraparte, se escucha, se pregunta, se es empático, se es prudente, se genera opciones de solución, no se pierde de vista las alternativas fuera de la mesa de negociación, se propone soluciones bajo criterios objetivos. Esta es una negociación colaborativa o de principios o de intereses (Harvard).

Hoy se suma a todas estas habilidades la necesidad de dominar las nuevas tecnologías, con el propósito de llevar adelante una sesión de negociación. No debe permitirse que el carente manejo de la tecnología ocasione un quiebre de la negociación. 
En la conciliación extrajudicial, el conciliador será quien se encargue de conducir la sesión; además de las habilidades desarrolladas para lograr un entendimiento entre las partes, también debe dominar las nuevas tecnologías. Tiene que ser capacitado en el uso de esta nueva plataforma virtual, ya que cualquier error podría ser materia de sanción administrativa por el MINJUSDH, según la normativa vigente.

Estamos frente a nuevos tiempos, hay que adecuarnos a esta nueva era digital; sin embargo, no debe dejarse de lado las habilidades de negociación, que servirán para negociar o conducir una sesión como conciliadores.

Avancemos sin perder de vista el objetivo: negociar.

\section{REFERENCIAS}

Bush, R. A., y Folger, J. (1996). La promesa de mediación. Cómo afrontar el conflicto mediante la revalorización y el reconocimiento. Barcelona: Ediciones Granica.

Fisher, R., Ury, W., y Patton, B. (1992). Getting to yes. Negotiating an agreement without giving in (2. ${ }^{a}$ ed.). Nueva York: Random House Business Books.

Goleman, D. (2019). ¿Qué es la empatía? Y por qué es importante. En Inteligencia emocional. Empatía (pp. 1-14). Boston, MA: Harvard Business Review.

Hernández, A. (2011). Negociar es fácil, si sabe cómo. ¿Cuánto dinero pierde por desconocer las técnicas de negociación? (13. a ed.). Barcelona: Alienta Editorial.

Malhotra, D. (2016). Negociar lo imposible. Cómo destrabar y resolver conflictos difíciles (sin dinero, ni fuerza). Barcelona: Empresa Activa.

Organismo Peruano de Consumidores y Usuarios. (30 de septiembre del 2020). Preocupante: Internet Perú fijo y móvil están $58 \%$ y $37 \%$ por debajo del promedio mundial en agosto. Recuperado de https://opecu.org.pe/2020/09/30/ opecu-preocupante-internet-peru-fijo-y-movil-estan-58-y-37-por-debajo-delpromedio-mundial-en-agosto/

Real Academia Española. (2020). Diccionario de la lengua española. Recuperado de https:// dle.rae.es/vinculante

Shell, R. (2005). Negociar con ventaja. Estrategias de negociación para gente razonable. Barcelona: Antoni Bosch Editor.

Voss, C. (2016). Rompe la barrera del no. Nueve principios para negociar como si te fuera la vida en ello. Barcelona: Conecta. 
